\title{
A Routing Algorithm Based on Hybrid Antenna for Wireless Sensor Networks in Large Area
}

\author{
Jiang-Peng Weng, Hua-Qiang Chen, Feng-Lian Tie \\ College of Electronic Engineering, South China \\ Agricultural University, China \\ E-mail: vmishiwjpv@163.com, chenhuaqiang@163.com \\ 65517386qq.com
}

\author{
Bao-Xia Sun \\ Guangdong Engineering Polytechnic, China \\ E-mail: sunbaoxia@126.com
}

\author{
Wei-Xing Wang \\ College of Electronic Engineering, South China Agricultural University, China \\ Guangdong Engineering Research Center for Monitoring Agricultural Information, China \\ Key Laboratory of Information Acquisition and Application in Agriculture, Guangzhou Science Technology and Innovation \\ Commission, China \\ E-mail: weixing@scau.edu.cn
}

\begin{abstract}
Periodically collecting information and then transmit to a remote base station (BS) is one of the most important applications of sensor networks. But the energy restriction of battery makes this difficult, especially in large area. In order to improve the lifetime of networks in large area, we propose EEHA (energy-efficient gathering algorithm for WSN with hybrid antenna) in this paper, a protocol that is based on hybrid networks which contain directional antennas and omnidirectional antennas. In EHAA, a threshold of distance is defined to avoid forming long chain and sensors are grouped into several chains. Sensors can send data to each other through omnidirectional antennas while they can communicate with BS through directional antennas [1]. Simulation results show that, compared to PEGASIS, EEHA increases the network stable period greatly and performs better by $13 \%$, $14 \%, 9 \%$ when $20 \%, 50 \%$ and $100 \%$ of nodes die.
\end{abstract}

Keywords-large area; wireless sensor network; PEGASIS; hybrid antennas; routing

\section{INTRODUCTION}

Wireless sensor networks (WSN) can be used for information acquiring and data processing in agricultural monitoring. It synthesizes the modern sensor technology, microelectronic technology, the embedded computation technology and the wireless communication technology, and is considered as one of the top 10 technology to change the world in 21st century. With the advantage of low power consumption, low cost and high reliability, WSN becomes more and more popular in agriculture [2,3,4]. Traditionally, most of the WSN are assumed to be equipped with omnidirectional antennas due to its simplicity. However, nodes with omnidirectional antennas are easy to die of long-distance communication when they are deployed in large area. The use of directional antennas can extend communication range with the same amount of energy for transmission while it can only work at a narrow angle $[5,6]$. Thus, it is significant to study the hybrid networks which contain directional antennas and omnidirectional antennas if we can comprehensively utilize the advantage of two sides
$[7,8]$.

In this paper, we propose EEHA, a multi-chain based protocol. Sensors are grouped into several chains. In each chain, one sensor is selected as a leader based on its position and its residual energy. All leaders send the information to the BS through directional antennas, while other nodes send data to neighbor nodes through omnidirectional antennas. Due to the multiple chains structure, EEHA balances the energy consumption and avoids forming long chain. Thus, it can save the amount of energy spent per round and prolong the network lifetime. It also reduces the communication delay.

\section{RELATED WORKS}

The PEGASIS protocol [9] is an elegant solution where a chain is formed including all nodes in the network using the greedy algorithm to reduce the transmitting distance. However, PEGASIS has several defects: 1) the inevitability of long link between neighboring nodes lead to rapid death of some nodes 2) the communication delay from the original node to the end node is intolerable in some cases 3) the leader nodes which are far from the BS expend great amounts of energy in one round 4) it will need to rebuild the whole chain when some nodes die.

Many new algorithms have been proposed to address the problems above. EEPB (Energy-Efficient PEGASIS-Based protocol) [10] adopt a threshold to decrease the formation of long link. In [11], author proposes a protocol based on LEACH [12] and PEGASIS. In the process of building the cluster, the protocol calculates the dispersion coefficient and determines cluster communication by the threshold. Cluster-head sends its data to gateway by single hop or multi-hop. Hu Jun-hao [13] uses tabu algorithm to optimize the topology. In addition, authors in [14,15] divide monitoring area into several areas. Routing tree is built in each area and leader nodes are elected to communicate with BS. COSEN (A Chain Oriented Sensor Network for 
Efficient Data Collection) [16] is a hierarchical chain based protocol. Sensors in the area are grouped into one higher level chain and several lower level chains. Simulation results show that COSEN requires much lower time and energy due to multiple chains and hierarchical structure.

\section{NETWORK AND RADIO MODEL}

In this paper, we consider the following network model assumptions:

1) The BS with sufficient energy is located far from the network.

2) All nodes are homogeneous and their location and energy information are available.

3) No mobility of sensor nodes

4) Each node has power control and the ability to transmit data to other sensor with omnidirectional antenna or to the BS with directional antenna.

We use the radio model described in [17]. If the distance is less than a threshold $d_{0}$, the free space is used; otherwise, the multipath model is used. To transmit a 1-bit message a distance $\mathrm{d}$ with, the radio expends:

$$
\begin{array}{r}
E_{T X}(l, d)=E_{T X-\text { elec }}(l)+E_{T X-a m p}(l, d) \\
=\left\{\begin{array}{l}
l * E_{\text {elec }}+l * \varepsilon_{f s} * \mathrm{~d}^{2}, d \leq d_{0} \\
l * E_{\text {elec }}+l * \varepsilon_{\mathrm{mp}} * \mathrm{~d}^{4}, d>d_{\mathrm{o}}
\end{array}\right.
\end{array}
$$

To receive this message, the radio expends:

$$
E_{R X}(k)=E_{R X-\text { elec }}(k)=l * E_{\text {elec }}
$$

In this model, the radios have power control so that they can expend the minimum required energy to reach the intended recipients.

When nodes use directional antennas to send information with same transmit power, the communication range $d_{\theta}$ is given by [18],

$$
d_{\theta}=k d_{o m n i}=\left(\frac{2}{1-\cos (\theta / 2)}\right)^{\frac{1}{2}} d_{o m n i}
$$

If $\theta=360^{\circ}$, then $d_{\theta}=d_{\text {omni }}$. Thus, to transmit an l-bit message a distance $d$ using directional antennas, the radio expends:

$$
\begin{array}{r}
E_{D T X}(l, d)=E_{D T X-\text { elec }}(l)+E_{D T X-a m p}(l, d) \\
=\left\{\begin{array}{l}
l * E_{\text {elec }}+l * \varepsilon_{f s} * \mathrm{~d}^{2} / k^{2}, d \leq k * d_{0} \\
l * E_{\text {elec }}+l * \varepsilon_{\mathrm{mp}} * \mathrm{~d}^{4} / \mathrm{k}^{4}, d>k * d_{0}
\end{array}\right.
\end{array}
$$

In the case of $\theta=60^{\circ}, \mathrm{k} \approx 3.9$.

\section{EEHAPROTOCOL}

The main idea in EEHA is to avoid forming long link by multiple chains structure and take advantage of directional antennas in long-distance transmission.

\section{A. CHAIN FORMATION PHASE}

In PEGASIS, the neighbor distance will increase gradually because nodes already on the chain cannot be revisited.

In order to reduce the formation of long link, EEHA defines a distance threshold D.

$$
D=\alpha E\left(d_{2 \text { next }}\right)
$$

where $\alpha$ is the parameter of long link and $E\left(d_{2 n e x t}\right)$ is the mathematical expectation of distance between neighbor nodes.

Like PEGASIS, we start with the furthest node from the BS to construct the chain. The furthest node finds its closest neighbor using greedy algorithm at first. This neighbor finds its next node in the same way. Assuming node $i+1$ is the closest neighbor of node $i$, EEHA compare the distance between node $i+1$ and node $i$ with $\mathrm{D}$ at first.

If $d i \leq D$, then the link between node $i+1$ and node $i$ is not a long link, and node $i+1$ will be linked to node $i$

Otherwise, the link between them is considered to be a long link which should be reduced if possible. At this time, new chain formation starts from node $i+1$. Repeat the above steps until all the nodes are visited. Several chains will be formed finally and the number of chains is determined by $\alpha$.

The value of $\alpha$ is important during the formation, and it will be discussed in the following part.

If there are 100 nodes in the network and they are grouped into 10 short chains, the average energy consumption of the whole network is given in Eq. 6 when a node sends information to next node using omnidirectional antennas.

$$
\begin{aligned}
E_{2 \text { next }} & =E_{T X}\left(l, d_{2 \text { next }}\right)+E_{R X}(l) \\
& =\left(l E_{\text {elec }}+l E_{f s} d_{2 \text { next }}^{2}\right)+(1-10 / 100) l E_{\text {elec }} \\
& =1.9 l E_{\text {elec }}+l E_{f s} d_{2 \text { next }}^{2}
\end{aligned}
$$

When a node sends information to BS using directional antennas, the average energy consumption of the whole network is:

$$
E_{2 \sin k}=E_{T X}\left(l, d_{2 \sin k}\right)=l E_{\text {elec }}+l E_{f s} d_{2 \sin k}^{2} / k^{2}
$$

Thus, a node is better to transmit to BS using a directional antenna than transmit to next node using an 
omnidirectional antenna when

$$
\Delta E=E_{2 \text { next }}-E_{2 \sin k}>0
$$

In the case of $k=3.9$,

$$
\Delta E=6.75 \times 10^{\wedge} 4+15 \times \mathrm{d}_{2 \text { next }}^{2}-d_{2 \sin k}^{2}>0
$$

To a network whose area is $\mathrm{M} \times \mathrm{M}$ and $\mathrm{BS}$ coordinate is $(\mathrm{x} 0, \mathrm{y} 0)$, the expected distance from nodes $(\mathrm{x}, \mathrm{y})$ to $\mathrm{BS}$ is given by

$$
\left\{\begin{array}{l}
\frac{1}{M^{2}} \iint\left(x-x_{0}\right)^{2}+\left(y-y_{0}\right)^{2} d x d y \\
0 \leq x \leq M, O \leq y \leq M
\end{array}\right.
$$

When $\mathrm{M}=500, \quad \mathrm{x} 0=250 \quad$ and $\mathrm{y} 0=600$, $\mathrm{E}\left(d_{2 \sin k}^{2}\right)=1.64 * 10^{\wedge} 5$; when the number of nodes $\mathrm{n}=100$, $\mathrm{E}\left(d_{2 \text { next }}\right)=50$; so the value of $\alpha$ should be about 1.6. In addition, the value of $\alpha$ should be larger in two cases: 1) the nodes of short chain are in the area which is far from BS, so the leader node is easy to die. 2) the death of some nodes lead to the average distance between neighbors become longer, so it is better to increase $\alpha$ to avoid forming too much short chains. In this paper, the value of $\alpha$ is set to 1.6. When the nodes in the chain are far from BS or $10 \%$ of nodes in the network are dead, the value of $\alpha$ increases to
2.

\section{B. LEADER ELECTION AND DATA TRANSMISSION PHASE}

Since the BS is located far away, the cost to transmit to the BS is high. Thus the nodes near BS should be elected leader. Besides, the nodes with less energy should not be elected leader in order to avoid rapid death of nodes. Therefore, the nodes which are near BS and have more energy get priority in the election of leader. In EEHA, the node with the smallest $\mathrm{R}$ is elected as a leader. The value of $\mathrm{R}$ is given by:

$$
R=d_{i} / E_{\text {res }}(i)
$$

where $d_{i}$ is the distance between node $i$ and BS, and $E_{\text {res }}(i)$ is the residual energy of node $i$.

After electing leaders, nodes in each short chain send data to their leaders as in PEGASIS. Every leader then transmits the information to BS using a directional antenna.

\section{SIMULATION RESULTS}

In our simulation, we use MATLAB R2014a as our simulator. We assume 100 nodes placed randomly in a $500 \times 500$ field. The nodes in the network have same energy

\begin{tabular}{|c|c|}
\hline Parameter & Value \\
\hline Network size $/ \mathrm{m}^{2}$ & $500 \times 500$ \\
\hline BS location & $(250,600)$ \\
\hline Node number $\mathrm{N}$ & 100 \\
\hline Initial energy of nodes $E_{0} / \mathrm{J}$ & 0.5 \\
\hline Electronics energy $E_{\text {elec }} /\left(\mathrm{nJ} \cdot \mathrm{bit}^{-1}\right)$ & 50 \\
\hline Amplifier consumed energy in free space model $\varepsilon_{\mathrm{fs}} /\left(\mathrm{pJ} \cdot \mathrm{bit}^{-1} \cdot \mathrm{m}^{-2}\right)$ & 10 \\
\hline Amplifier consumed energy in multipath model $\varepsilon_{m p} /\left(\mathrm{pJ} \cdot \mathrm{bit}^{-1} \cdot \mathrm{m}^{-4}\right)$ & 0.0013 \\
\hline Transmit threshold $d_{0} / \mathrm{m}$ & 87.7 \\
\hline Packet size $l$ /bits & 4000 \\
\hline Parameter of long link & 1.6 \\
\hline
\end{tabular}
at the beginning. Table 1 shows the parameters used in simulations.

TABLE I. THE PARAMETERS USED IN SIMULATIONS 


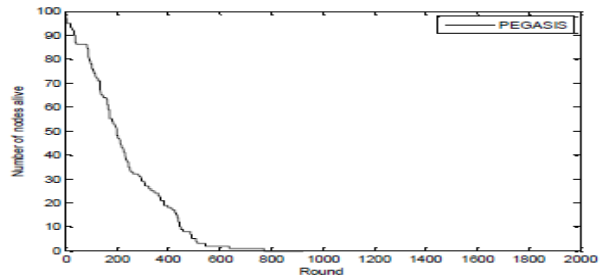

Figure 1. Lifetime of PEGASIS with omnidirectional antennas.

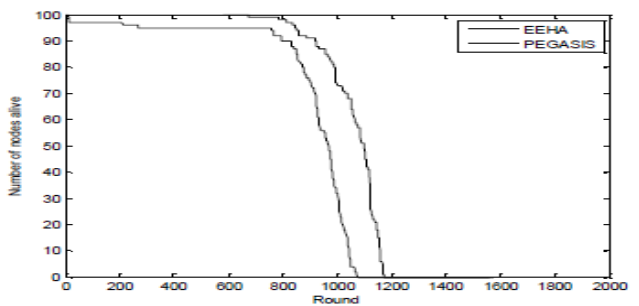

Figure 2. Lifetime of EEHA and PEGASIS with hybrid antennas.

Since the area is large and BS is far away from the network, the transmit distance is long. When nodes in the network are only equipped with omnidirectional antennas, they will die very quickly for the high cost of energy. When nodes in the network are equipped with directional antennas and omnidirectional antennas, EEHA shows better performance than PEGASIS in terms of energy consumption and transmission delay. Fig. 1 and Fig. 2 shows the network lifetime of PEGASIS and EEHA. The first node dies at 673 rounds for EEHA, whereas the first node dies for PEGASIS at 10 rounds. Compared to PEGASIS, EEHA increases the network stable period greatly and performs better by $13 \%, 14 \%, 9 \%$ when $20 \%$, $50 \%$ and $100 \%$ of nodes die. Fig. 3 shows the residual energy of nodes in the network.

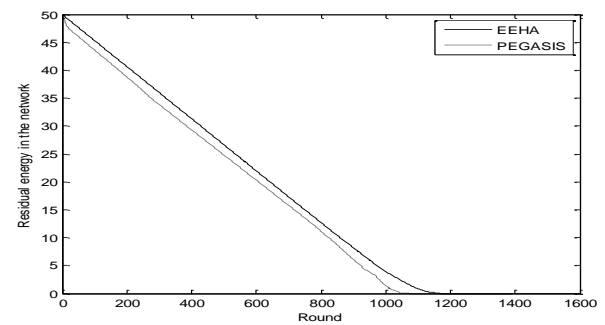

Figure 3. Residual energy comparison.

\section{CONCLUSION}

In this paper we propose EEHA, a multi-chain protocol based on hybrid antennas for large area. Nodes in the network can send information to BS through directional antennas or to neighbor nodes through omnidirectional antennas. They are grouped into several short chains. A leader is elected in each chain and it gathers data before transmitting to the BS. EEHA outperforms PEGASIS by reducing the formation of long link, using directional antennas in long-distance transmission, optimizing the election of leaders. Simulations show that EEHA prolongs the network lifetime and increase the network stable period.

\section{ACKNOWLEDGEMENT}

This research was financially supported by Natural Science Foundation of Guangdong Province, China (2014A030313451); Science and Technology Planning Project of Guangdong Province, China (2015A020209013); Science and Technology Planning Project of Guangdong Province, China (2015A020209161)

\section{REFERENCES}

[1] Bettstetter C, Hartmann C, Moser C. How does randomized beamforming improve the connectivity of ad hoc networks? 2005

[2] Shi Fei. Research on routing algorithm for wireless sensor network based on energy balance. XI'AN technological university, 2010.

[3] Qu Huaxiang, Zhao Ping,Chen Guipeng, et al Advances in precision agriculture-based wireless sensor networks. Chinese Agricultural Science Bulletin. 2014(33): 268-272.

[4] Han Wenting, Wu Pute, Yu Xiaoqing, et al. Research progress in wireless sensor network for agricultural environment monitoring. Transactions of the CSAE. 2011(S2): 326-330

[5] Cho J, Lee J, Kwon T, et al. Directional Antenna at Sink (DAaS) to prolong network lifetime in Wireless Sensor Networks. 2006.

[6] Feng Ping. Research on anypath routing in wireless networks with directional antennas. Shanghai Jiao Tong University, 2013.

[7] Lei Gang, Wang Weixing, Sun Baoxia, et al. Design and optimization of wireless sensor networking in big paddy field. Transactions of the Chinese Society of Agricultural Engineering. 2014(11): 180-187.

[8] Sun Baoxia, Wang Weixing, Lei Gang, et al. Energy-efficient clustering algorithm based on hybrid antenna for heterogeneous wireless sensor networks. Computer Engineering and Design. 2014(08): 2851-2855.

[9] Lindsey S, Raghavendra C S. PEGASIS: Power-efficient gathering in sensor information systems. 2010.

[10] Yu Yongchang, Wei Gang. An improved PEGASIS algorithm in wireless sensor network. Acta Electronica Sinica. 2008, 36(7): 1309-1313.

[11] Li Jianqi, Cao Binfang, Wang Li, et al. Improved routing protocol in wireless sensor based on LEACH and PEGASIS. Chinese Journal of Sensors and Actuators. 2012(02): 263-267

[12] Heinzelman W. Energy-efficient communication protocols for wireless microsensor networks. 2000:3005-3014.

[13] Hu Junhao, Liu Xingchang, Tan Zuofei. An improved scheme based on PEGASIS algorithm. Microelectronics and Computer. 2014(11): 36-40.

[14] Liu Weiqiang, Jiang Hua, Wang Xin. Research and improvement of PEGASIS protocol in wireless sensor network. Chinese Journal of Sensors and Actuators. 2013(12): 1764-1769.

[15] Li Shuyan, Li Layuan. PEGASIS protocol for wireless sensor networks. Journal of WUT(Information and management engineering). 2012(04): 426-429.

[16] Mamun Q E K M, Urano Y. COSEN: A Chain Oriented Sensor Network for Efficient Data Collection. 2006.

[17] Heinzelman W B, Chandrakasan A P, Balakrishnan H. An application-specific protocol architecture for wireless microsensor networks. IEEE Transactions on Wireless Communications. 2002 , 1(4): 660-670.

[18] Hu Qingsong, Wu Lixin, Zhang Shen, et al. Balanced energy-efficient routing algorithm based on smart antenna and dynamic virtual cluster. Journal on Communications. 2013(8): 169-176.

[19] Lu Fei. Research on an Improved Distance Threshold PEGASIS Protocol for Wireless Sensor Network. East China Normal University, 2012. 